\title{
SHUT UP I'M DRIVING! IS TALKING TO AN INCONSIDERATE PASSENGER THE SAME AS TALKING ON A MOBILE TELEPHONE?
}

\author{
Natasha Merat, A. Hamish Jamson \\ Institute for Transport Studies \\ University of Leeds \\ Leeds, United Kingdom \\ E-mail: N.Merat@,its.leeds.ac.uk
}

\begin{abstract}
Summary: The objective of this study was to compare driving performance whilst talking on a hands-free mobile (cellular) telephone with performance during conversations with "considerate" and "inconsiderate" passengers. Using the Leeds Advanced Driving Simulator, participants were asked to drive through a road containing four driving scenarios: (1) car following along a straight road section, (2) car following along a curved section of road, (3) a braking event, and (4) a coherence event. A working memory digit recall and sentence verification task were used to simulate conversation in three conversation conditions: (1) "considerate passenger," where the experimenter asked drivers to responsd to the working memory task before and after a driving event, (2) "inconsiderate passenger," where the experimenter asked drivers to responsd to the working memory task throughout a driving event, and "3) "mobile phone task," which involved digital presentation of the working memory task via a mobile telephone throughout the driving events. A silent condition was also used as control. The effect of the three conversation conditions on driving performance was the same during the simple car following scenarios. However, talking to an "inconsiderate" passenger was found to be as disrupting as a mobile phone conversation, and different from conversing with a considerate passenger, during the braking and coherence conditions. Therefore, the high workload imposed by conversation was only detrimental during the more difficult driving conditions, when demand for central attentional resources from both tasks was at its highest.
\end{abstract}

\section{INTRODUCTION}

Talking on a mobile phone whilst driving is thought to be associated with a four-fold increase in accident risk (Redelmeier \& Tibshirani, 1997). For example, a high proportion of rear-end collisions are associated with mobile phone use during a drive (Wilson, et al., 2003). Experimental studies on mobile phone use and driving have shown increased reaction time to events, sometimes leading to a total failure to notice important driving-related information (e.g., Hancock, Lesch \& Simmons, 2002; McKnight \& McKnight, 1993; Strayer \& Johnston, 2001).

The use of hand-held mobile telephones whilst driving was banned in the UK in December 2003. To clarify the conditions of this ban, guidelines published by the UK Department for Transport on mobile telephones and driving state that "pushing buttons on a phone while it is in a cradle or on the steering wheel... is not covered by the new offence, provided you don't hold the phone." The average UK citizen is likely to be aware of this ban, although it is debatable whether they are aware of further notes in this guideline, which state that: "hands-free phones are also distracting and you still risk prosecution for failing to have proper control of a vehicle." Indeed, many recent studies have shown that manual interactions such as holding the telephone and dialing a number are not the only disrupting factors, and that the verbal content of the conversation itself can also be disrupting. For example, Strayer and Johnston (2001) found that 
the same number of traffic signals was missed by participants when they were engaged in a hand-held mobile conversation as when they were talking on a hands-free mobile telephone. These authors state, however, that the content of the conversation is a more important factor when studying driving impairment, and report more disruptions during a difficult wordgeneration task, compared to a simple shadowing task. Similar results were found by McKnight and McKnight (1993), who report a higher proportion of missed traffic signals during a difficult problem-solving task, compared to simple "chit chat." The effort associated with a telephone conversation during driving is shown by studies that report an increase in subjective workload scores (Alm \& Nilsson, 1995; Brookhuis, de Vries \& de Waard, 1991), as well as a rise in physiological measures such as heart rate (Brookhuis, et al., 1991).

Fix (2001) argues that the use of a mobile telephone is no more demanding than other drivingindependent secondary tasks, such as eating, drinking, talking to a passenger or listening to the radio. However, this argument is partly disputed by Consiglio, et al. (2003), who found that passenger and telephone conversations increased brake reaction time equally and to a larger extent than listening to music or tuning a radio. These authors used a series of basic questions in their study in an attempt to simulate a relaxed conversation between the passenger and driver. Whilst the same pace of conversation was maintained in the "mobile" and "passenger" conditions, it can be argued that this simple form of conversation was perhaps not difficult enough to affect brake reaction time. Also, the brake reaction time task itself required response to a red lamp in a laboratory-based mockup of driving. Therefore, the absence of an interaction with other driving-related information such as traffic signals, additional cars and road users meant that subjects completed quite a simple driving task, which clearly left adequate attentional resources to cope with the simple conversation task. The comparable effect of passenger and mobile telephone conversations on driving-related performance has also been reported by Nunes and Recarte (2002), who found similar reaction time to visual secondary tasks during a drive along a Spanish highway. However, since these authors fail to provide an account of the traffic conditions in their field experiment, it is difficult to establish how much workload was imposed by the driving task. Also, while they report an affect of difficult conversations on the processing of visual stimuli in the driving scene, they fail to give an account of drivers' performance in the conversation tasks. It is therefore not clear whether conversation was suspended at any stage of the drive, or whether conversation performance was the same for remote and in-car conditions. Clearly, any pauses in conversation would have facilitated driving performance.

Research in other, non-driving, contexts has shown that, compared to face-to-face conversations, verbal interactions are slow and perceived to be more difficult when participants are unable to see one another (Alibali, Heath \& Myers, 2001). Within the driving domain, recent research has shown a difference in level of conversation between remote and in-car conditions (Crundall, et al., in press). Here, participants were recruited in pairs, and were asked to take part in conversations, either as drivers or passengers. Crundall et al. showed that conversation pace was significantly reduced in more difficult urban driving, while no difference in conversation was observed in the less demanding rural driving conditions. This change in conversation was observed in both the driver and (to a lesser extent) the passenger. However, drivers did not reduce their conversation pace when the conversation was with a remote passenger located elsewhere. Another interesting observation by Crundall and colleagues' study is that the driver's conversation pace was reduced by the mere presence of the passenger in the car, even when this passenger was blindfolded and unaware of the visual scene. Fairclough et al. (1991) suggest that 
remote conversations on the mobile telephone are problematic because of the psychological distance perceived by drivers in this situation. It can also be argued that mobile phone conversations are more disrupting to drivers because the speaker on the other side of the phone is not aware of the driving environment, and demands the driver's attention regardless of traffic conditions (Crundall, et al., in press; Gugerty, Rakauska \& Brooks, 2004; Parkes, 1991). As shown by Crundall et al., when confronted with a busy traffic situation, in-car passengers reduce the number and length of their speeches.

To summarize, studies that have shown similar effects on driving from talking on the mobile phone and in-car passengers have either used relatively simple driving tasks, and/or failed to report performance in the conversation task. Crundall et al. have shown that drivers do indeed reduce the intensity of their conversation during more demanding driving conditions, although they fail to give an account of driving performance measures such as speed of travel, degree of lateral deviation and so on. The objective of the experiment reported here was to examine performance in both driving and the conversation task. The interaction between a difficult conversation task and increasing levels of driving complexity was therefore examined.

Furthermore, the pace and level of conversation in the passenger condition was the same as the "mobile phone" condition, with both presented in the same manner during the difficult driving events. This "inconsiderate passenger" condition was compared to situations where the passenger was silent during the difficult driving events, speaking only before and after these sections.

\section{METHOD}

\section{Participants}

Twenty-four students (12 men and 12 women) from the University of Leeds were recruited for this study. They had a mean age of 20.9 years, and their driving experience ranged between 1.5 and 7 years.

\section{Design and Procedure}

Using the Leeds Advanced Driving Simulator, participants drove a simulated section of road containing four car following driving events, separated by "filler sections" (Figure 1). It was hypothesised that driving the straight and curve events would be less challenging than the braking and coherence events. This road layout was repeated 4 times for each participant.

Participants were required to complete one of four conversation conditions for each repetition of the road layout, and the order of these conversation conditions was counterbalanced across participants. The "conversation" consisted of a digit recall task, followed immediately by a reasoning task (see Baddeley, 1986).

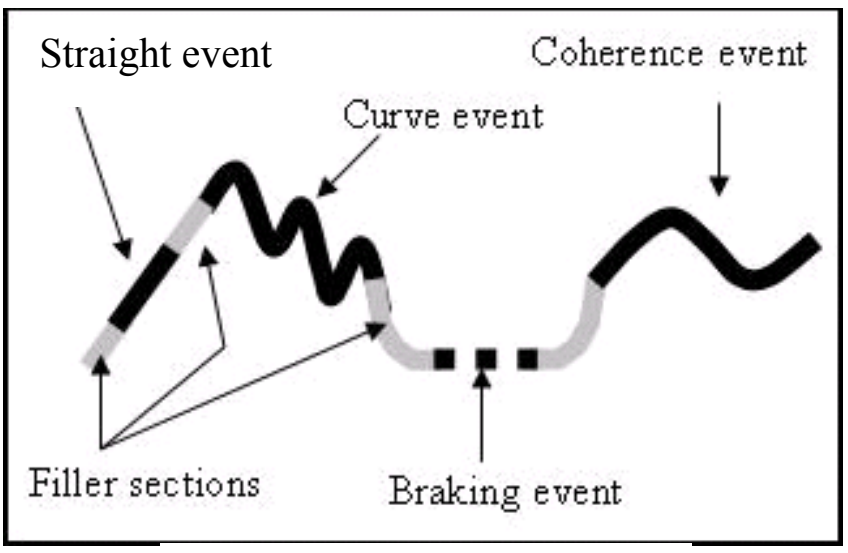

Figure 1. Road layout 
Participants were asked to respond to the reasoning task and then recall the digits in the correct presentation order. The reasoning task required a true/false response to statements such as ' $A$ is preceded by $B-B A^{\prime}$ ' (false). This highly controlled, demanding conversation task was presented to subjects in one of three ways: (i) in the "mobile phone" condition the digits and reasoning task were prerecorded and presented digitally from a PC during the event sections (ii) in the "inconsiderate passenger" condition, the numbers and digits were again presented during the events, but this time by the experimenter, who sat in the passenger seat, and (iii) in the "considerate passenger" condition, the numbers and digits were presented in the filler sections by the experimenter, who was silent during the event sections. The rate of digit presentation was controlled by the experimenter, using a metronome. A fully silent condition was also used with no conversation taking place in either the filler or event driving sections (see Table 1). As well as investigating the effect of these conversation conditions on driving behaviour, participants' performance in the digit recall and reasoning task was also examined.

Table 1. The placement of the conversation task in each condition

\begin{tabular}{|l|c|c|c|c|c|c|c|c|}
\hline Conversation & Filler & Straight & Filler & Curve & Filler & Braking & Filler & Coherence \\
\hline Silent & - & - & - & - & - & - & - & - \\
\hline Mobile & & task & & task & & task & & task \\
\hline Inconsiderate & & task & & task & & task & & task \\
\hline Considerate & task & & task & & task & & task & \\
\hline
\end{tabular}

\section{RESULTS}

Speed of travel was not affected by conversation type in the straight and curved road sections. In the straight driving event, standard deviation of lane position was the only driving measure that was affected by concurrent conversation $(\mathrm{F}(1,23)=5.96, \mathrm{p}<.01)$. Post-hoc t-tests showed less deviation in lane during the conversation conditions than the silent condition. This effective "improvement" in lateral control of the car by a demanding secondary task has been associated with a "narrowing of visual resources" towards the centre of the road, and is thought to be problematic for peripheral hazard detection (e.g., Brookhuis et al., 1991; Jamson \& Merat, 2005.)

During the braking event, minimum headway to the car in front was found to be significantly reduced for the "mobile telephone" and "inconsiderate passenger" conversation conditions, compared to the "silent" and "considerate passenger" conditions $(\mathrm{F}(1,23)=$ $3.47, \mathrm{p}<.05$, see Figure 2 ). Therefore, the effect of reduced attentional resources imposed by the conversation task had clear safety

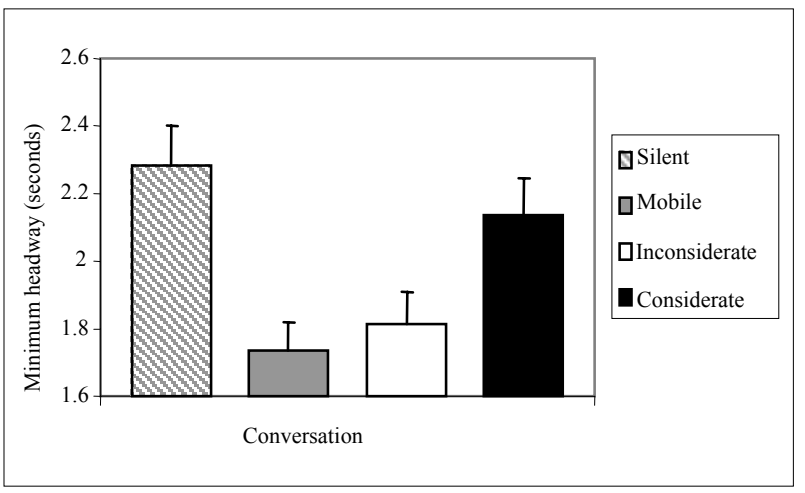
implications in the relatively difficult braking condition. 
The measurement of modulus in the coherence event provides information about the degree of "overshoot" or "undershoot" between the participants' speed pattern with respect to the lead car. If a driver is able to attend to the speed of the lead car, the modulus score will be close to 1 . This measure was found to vary significantly across the four conversation conditions $(\mathrm{F}(1,23)=$ $442.18, \mathrm{p}<.01)$. Post hoc t-tests showed a significant difference in modulus between the "silent" and "inconsiderate passenger" condition, the "silent" and "mobile telephone" condition, and the "inconsiderate" and "considerate passenger" conditions (see Figure 3).

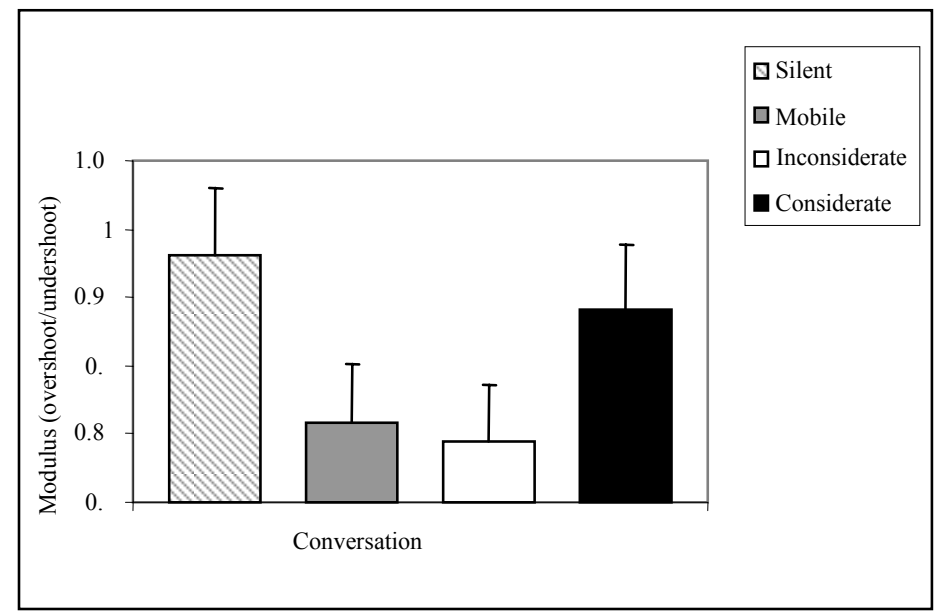

Figure 3. Modulus in the coherence event

Performance in the "inconsiderate passenger" and "mobile telephone" conversation tasks was analysed by counting the proportion of items recalled in the correct order (serial recall), the proportion of digits recalled from the list, regardless of position (item recall), as well as counting item errors, order errors and omissions. The "considerate passenger" condition was not included in the analysis since this conversation took place during the filler road sections.

Item recall was found to be affected by road category, with more digits recalled during the easier straight and curve car following sections $(\mathrm{F}(3,60)=$ $3.736, \mathrm{p}<.05)$. The least number of items were recalled during the braking event (see Figure 4). Serial recall was not different for the four road conditions. The proportion of "additional" digits not on the original list was found to be significantly higher in the coherence condition, compared to all other driving conditions $(\mathrm{F}$ $(3,60)=4.769, \mathrm{p}<.01$, see Figure 5).

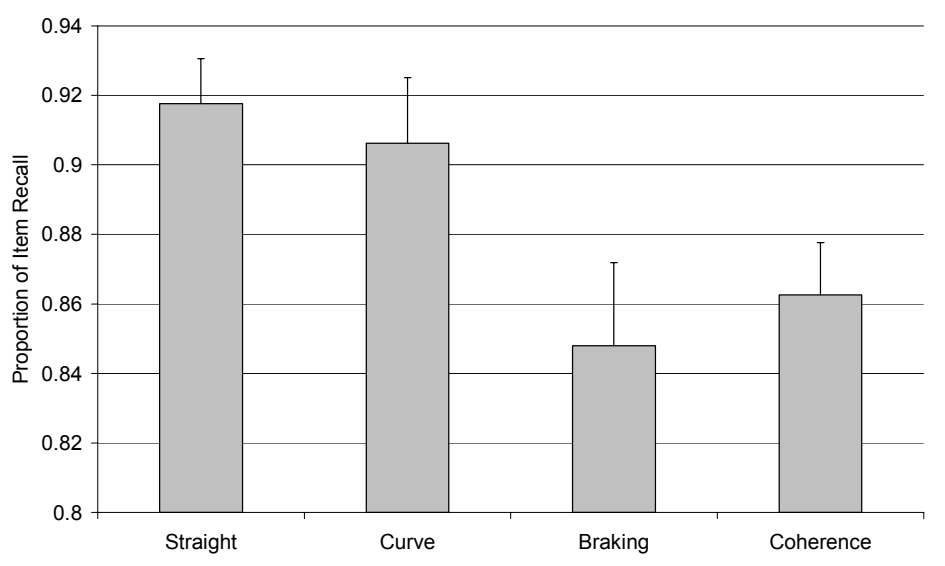

Figure 4. Item recall during the four driving conditions (mobile and inconsiderate combined) 


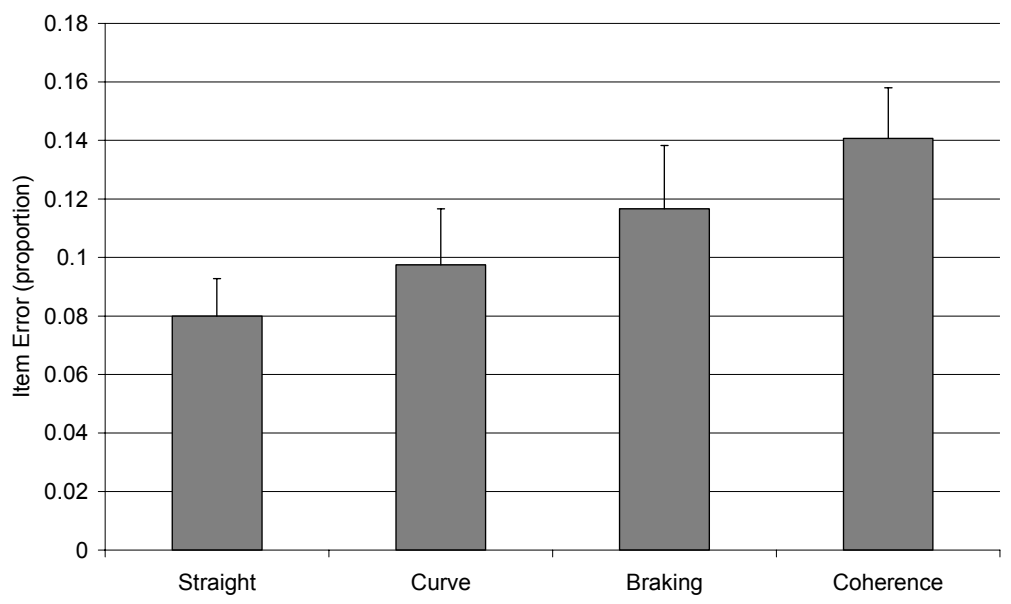

Figure 5. Item errors during the four driving conditions (mobile and inconsiderate combined)

\section{DISCUSSION AND CONCLUSIONS}

Impairments in the driving performance measures, and interruptions in the conversation task, were both found to be greatest during the more difficult driving conditions. These findings support the suggestion that mobile telephone conversations are most disruptive in difficult driving conditions, and especially when the attention demand from the telephone conversation itself is also quite high (e.g., Breim \& Hedman, 1995). The extent to which the conversation task affected driving was somewhat disappointing, although we believe that this may have been partly because this study used a relatively young group of drivers. Studying the performance of middleaged and older drivers in this task will therefore be interesting. Finally, while an attempt was made to vary difficulty of driving by using a braking and coherence event in the car following task, it is questionable whether these events were difficult enough to load drivers' attentional resources to their maximum capacity. Future research hopes to study the effect of these conversation tasks in an urban environment.

\section{ACKNOWLEDGMENTS}

The authors would like to thank Debbie Brew for her help in conducting this study, and Anthony Horrobin for creating the simulator scenarios.

\section{REFERENCES}

Alibali, M., Heath, D. \& Myers, H. (2001). Effects of visibility between speaker and listener on gesture production: some gestures are meant to be seen. Journal of Memory and Language, 44: 169-188.

Alm, H., Nilsson, L. (1995). The effects of a mobile telephone task on driver behaviour in a car following situation. Accident Analysis and Prevention, 27(5): 707-715.

Baddeley, A.D. (1986). Working Memory. Oxford: Clarendon Press. 
Breim, V. \& Hedman, L.R. (1995). Behavioural effects of mobile telephone use during simulated driving. Ergonomics, 38: 2536-2562.

Brookhuis, K.A., De Vries, G. and De Waard, D. (1991). The effects of mobile telephoning on driving performance. Accident Analysis and Prevention, 23: 309-316.

Consiglio, W., Driscoll, P., Witte, M. \& Berg, W.P. (2003). Effect of cellular telephone conversations and other potential interference on reaction time in a braking response. Accident Analysis and Prevention, 35(4): 495-500.

Crundall, D., Bains, M., Chapman, P. \& Underwood, G. (In press). Regulating conversation during driving: a problem for mobile telephones? Transportation Research Part $F$.

Fairclough, S.H., Ashby, M.C., Ross, T. \& Parkes, A. (1991). Effects of hands free telephone use on driving behaviour. In: Proceedings of the ISATA conference, Florence, Italy.

Gugerty, L., Rakauskas, M. \& Brooks, J. (2004). Effects of remote and in-person verbal interactions on verbalization rates and attention to dynamic spatial scenes. Accident Analysis and Prevention, 36: 1029-1043.

Hancock, P.A., Lesch, M. \& Simmons, L. (2002). The distraction effects of phone use during a crucial driving maneuver. Accident Analysis and Prevention, 35(4): 501-504.

Jamson, A.H. \& Merat, N. (2005). Surrogate In Vehicle Information Systems and Behaviour: Effects of Visual and Cognitive Load in Simulated Driving. Transportation Research Part F: Traffic Psychology and Behaviour.

McKnight, A.J. \& McKnight, A.S. (1993). The effect of cellular phone use upon driver attention. Accident Analysis and Prevention, 25(3): 259-265.

Nunes, L. \& Recarte, M.A. (2002). Cognitive demands of hands-free-phone conversation while driving. Transportation Research Part F, 5: 133-144.

Redelmeier, D.A. \& Tibshirai, R.J. (1997). Association between cellular-telephone calls and motor vehicle collisions. New England Journal of Medicine, 336: 453-458.

Strayer, D.L. \& Johnston, W.A. (2001). Dual task studies of simulated driving and conversing on a cellular telephone. Psychological Science, 12(6): 462-466.

Strayer, D.L., Drews, F.A. \& Johnston, W.A. (2003). Cell phone-induced failures in visual attention during simulated driving. Journal of Experimental Psychology: Applied, 9(1): 23-32.

Wilson, J., Fang, M. Wiggins, S., \& Cooper, P. (2003). Collision violation involvement of drivers who use cellular telephones. Traffic Injury Prevention, 4: 45-52. 